\title{
MasePlanner: A Card-Based Distributed Planning Tool for Agile Teams
}

\author{
Robert Morgan and Frank Maurer \\ Department of Computer Science, University of Calgary \\ Calgary, Alberta, Canada, T2N 1N4 \\ \{morganr, maurer\}@cpsc.ucalgary.ca
}

\begin{abstract}
Supporting agile teams from afar as they plan projects is a challenge. In this paper, we present a planning tool, MasePlanner, which supports agile teams as they collaborate in a distributed environment during their planning meetings. Combined with an audio link, our tool supports natural interaction similar to those found in collocated agile planning environments. MasePlanner supports planning in both vertical and horizontal tabletop workspaces by allowing participants to use interaction techniques such as moving and piling of story cards. By implementing a strong index-card metaphor, the tool aims to be more closely aligned with natural environments
\end{abstract}

\section{Introduction}

In Extreme Programming (XP) [1], communication is strongly encouraged throughout the various activities of the development process. Effective communication with offsite team members and customers is often a challenge for distributed development teams. In this paper, we focus on agile project planning and tool support for synchronous distributed planning. Project

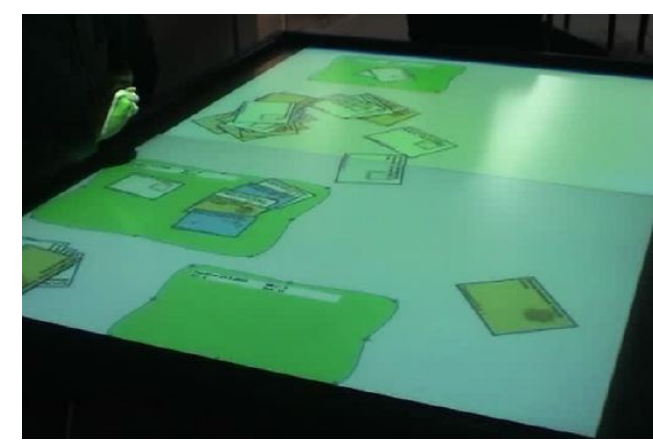

Figure 1. AgilePlanner running on a digital tabletop display. planning in an agile project involves communicating and collaborating with all members of the team at once. When working in a distributed setting, this poses the challenge of communicating the creation and organization of information generated to all participants.

Communication in agile project planning is important to facilitate shared knowledge and understanding of the direction of the project. Agile teams use cards to represent user stories/backlog entries/feature requests/tasks as steps towards the completion of the project. Using pen and index cards, collocated teams are able to quickly create cards and organize them for all to see. With non-collocated teams this card-based planning is not possible as team members are not able to physically share the cards created. To overcome this issue, several web-based systems were developed in the past that support knowledge sharing and agile planning in distributed teams ${ }^{1}$. Existing software solutions primarily are using forms and tables to edit and display planning information on a web page. Compared with paper index cards, the interaction with these tools is awkward and slow and they are limiting the efficiency of teams in planning sessions. Our (informal) observations of agile teams indicate that collocated teams often use index cards during planning meetings and then later transcribe them into web-based tools for progress tracking. As this seems to be a waste of time, we are now investigating innovative user interface metaphors for overcoming these issues and provide teams a planning experience that matches card-based planning as closely as possible.

Tool support should allow for creating and organizing cards during the meeting and not post meeting. Agile planning tools need to support the creation and organization of story cards in a natural way similar to collocated settings. This suggests that

\footnotetext{
1 Our own MASE system [2] was one of the first. Now several commercial (e.g. [3, 4]) and open source tools (e.g. [5]) are available.
} 
digital cards be easy to create, organize and destroy akin to their physical counterparts. MasePlanner is an agile planning tool that supports natural interactions for card based planning facilitating non-verbal communication. In this short paper, we provide an overview on its functionality and anticipated benefits. An empirical investigation will be conducted later and is currently beyond the scope of this paper.

The paper is organized as follows: Section 2 describes the project planning process and the interactions that need to be supported; Section 3 presents related works supporting planning in agile teams; Section 4 describes the tool and its implementation; Section 5 describes planning with MasePlanner; Section 6 discusses proposed methods of evaluation and future plans. We conclude the paper with a summary of our work to date in Section 7.

\section{Agile Planning - An Overview}

Agile planning meetings are an opportunity for both the customer and the developers to come together and discuss the future of the software project [1]. Teams use stories to provide a high level description of customer requirements for the project. Index cards are used for documenting the stories as they allow for easy creation and organization. The combination of verbal communication and physical organization of the story cards allows for all involved to understand the direction of the project. Organizing the cards on large surfaces like tables and whiteboards [6] helps teams to better understand the direction of the project.

Developers estimate the effort for story cards created during the planning meeting [1]. Time estimations are made for the best, worst, and most likely amount of time that the given story will take to complete. Customers then prioritize cards based on what they believe will provide the most value to the project. Once stories are estimated and prioritized they are ready to be organized into iterations. Iterations refer to the fixed time between the current planning meeting and the next planning meeting. This time frame will typically vary from one to four weeks depending on the project. The organization of story cards into iterations is highly dependent on their time estimates, the time available and the priority that they have been given [1].

The organization of cards into an iteration is often extended to include subsequent iterations. Cards that are not placed into the iterations are stored in a product backlog. Piling of cards together is often combined with their physical location on a table to indicate iterations or the product backlog [6]. Organizing story cards is a highly interactive process, with cards being created, edited, repositioned and even discarded with ease and, partially, concurrently by multiple team members.

\section{Related Work}

Tools for supporting agile planning are now widely available. These tools allow for sharing of planning information between team members. Many of the existing planning tools are web based making use of forms and tables to represent planning information and its organization. The common limitation that these tools possess is there inability to support true cardbased planning and natural interactions.

Existing tools like $[3,4,5,7,8]$ allow for cards to be created and organized into iterations. These tools limit the amount of collaboration possible because they do not provide the spatial awareness created by the physical location of objects that exist in a traditional planning environment. These tools provide only one keyboard/mouse for a shared display resulting in limited concurrent work and hence requires much more turn-taking than a "normal" agile planning meeting. This often results in teams using index card based planning approaches during the planning meetings and then transcribing the results into the planning tool. These tools are then used for data management and progress tracking following the meeting.

As most existing planning tools are web based, they fall into the one-computer/one-user paradigm [6]. This additionally contributes to their use as information sharing and management systems rather then true collaborative applications.

\section{MasePlanner}

MasePlanner is the next step in providing agile teams a planning environment that meets the need of collocated and distributed teams. The MASE system described in [2] took steps to provide team members access, and the ability, to create project information regardless of their location. We later extended this work with the development of AgilePlanner [6,9].

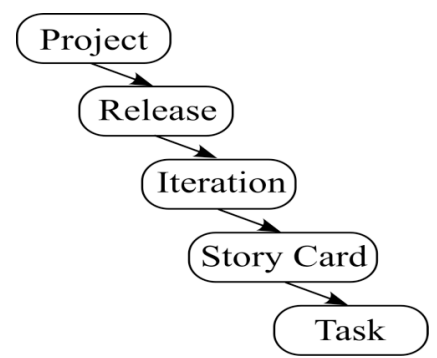

Figure 2. Supported planning hierarchy 


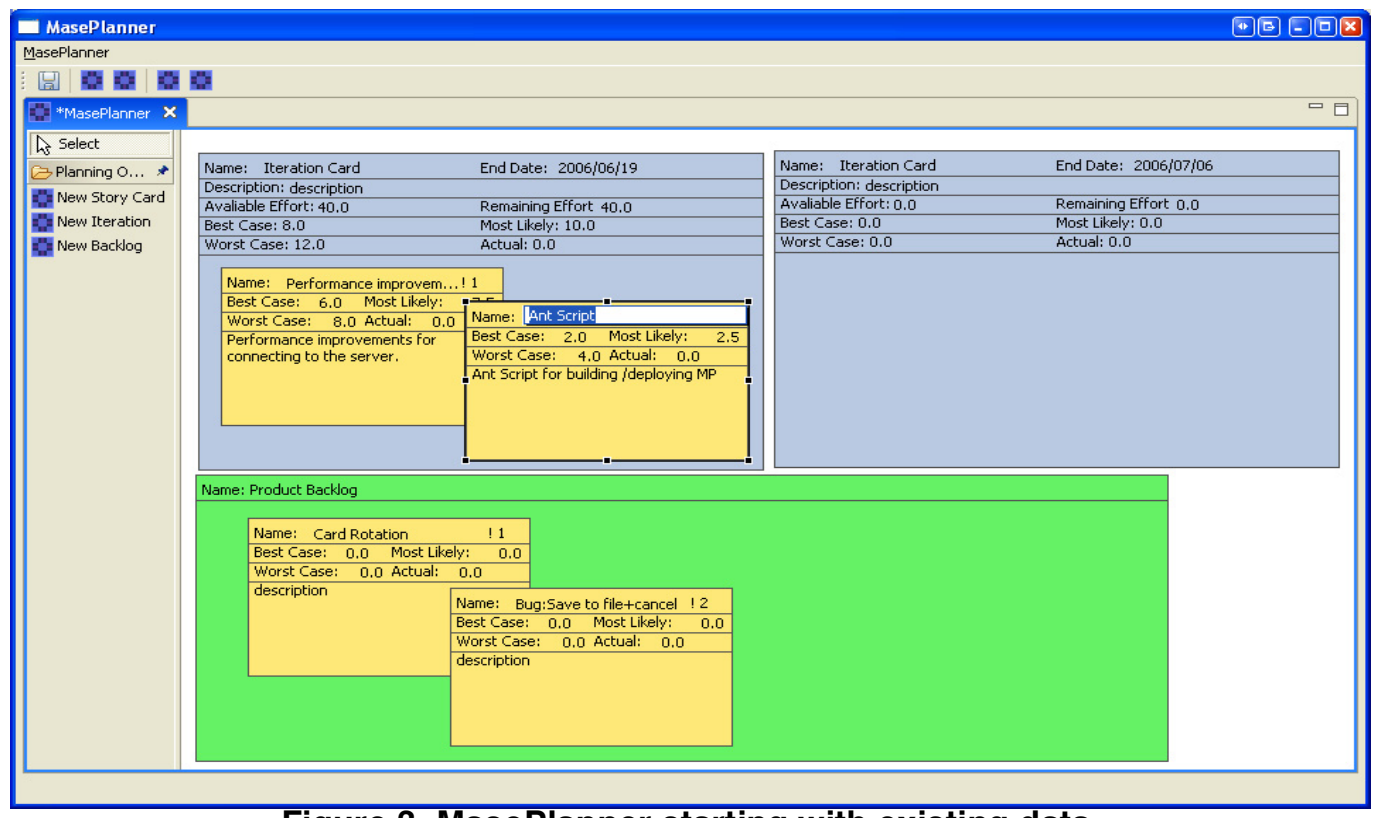

Figure 3. MasePlanner starting with existing data.

AgilePlanner examined the impact of card-based planning in a collocated digital tabletop environment (Figure 1). AgilePlanner extended existing planning tools by allowing for collocated teams to create and organize planning artifacts through the use of tablet PC's and a digital tabletop surface [6,9]. Through a formal evaluation of AgilePlanner, important conclusions were highlighted. The handwriting recognition and the ability to organize artifacts similarly to paper based environments were determined to be important. Identified areas for improvement, were the creation of planning artifacts, story cards estimation and prioritization. These were taken into account when developing our new approach.

\subsection{MasePlanner}

MasePlanner is developed to improve the planning process by providing teams with a digital environment that supports information management in addition to natural interactions. Regarding support for natural interaction, MasePlaner allows for planning artifacts to be created, edited, and organized in a similar manner to paper based planning meetings.

Similar to existing tools, MasePlanner supports the creation of story cards and iterations allowing for their organization. It extends this by allowing card-based planning which provides spatial awareness of the cards location. MasePlanner is designed as a support tool and as such requires that there exists a high quality voice channel for true collaboration to take place.

MasePlanner is designed to fit into a client server environment. The application depends on existing servers to retrieve and store all of its planning data. MasePlanner is not limited to supporting one specific server and can be extended to connect to various systems. The limitation to extending MasePlanner is that the hierarchy depicted in Figure 2 has to be supported by the planning server. Currently, MasePlanner has been extended so it is able to connect to our Mase [2] server as well as to connect to the Rally [4] server.

MasePlanner is implemented as an eclipse plug-in [10] with web services support for remote connectivity. To allow for natural interactions, we made use of the graphical editing framework (GEF) [11] as this facilitates both the graphical representation and spatial organization of planning artifacts.

\section{Planning with MasePlanner}

MasePlanner supports planning meetings by providing a common workspace similar to a tabletop for story cards to be created and organized on. With regards to existing projects, MasePlanner will load story card data and recreate the layout that previously existed (Figure 3). This provides all participants, regardless of location, access to the environment as if they were collocated. 


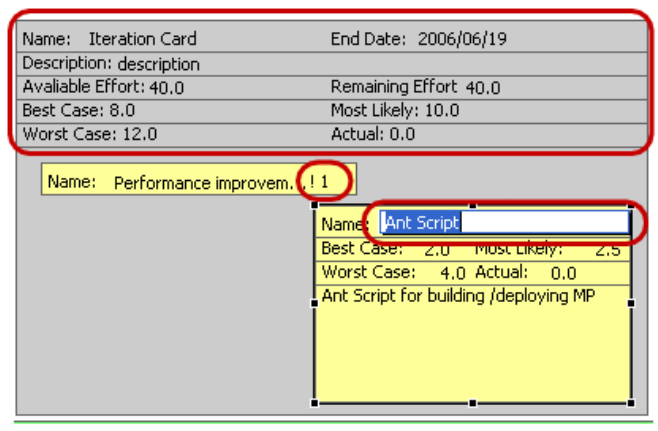

Figure 4. Story card priorities, Iteration summarv and direct edit

The interface layout provides quick access to widgets to create story cards, iterations, and backlog regions to the left of the collaborative workspace. Editing of the story cards is currently done by directly editing the text on the various planning objects ${ }^{2}$. The workspace, unlike a physical tabletop workspace, will expand to fit all the story cards.

Screen real estate can be of concern to some team members. To allow for a portion of the workspace to be recovered, story cards can be minimized in place. This allows for more cards to be viewed, in a limited space, without having to see the details of the card (Figure 4).

\subsection{Card Creation}

Story card creation, as we saw in Section 2, is an important aspect of agile planning. Using MasePlanner, cards are easily created by clicking the story card widget and then clicking on the location where the card is to be placed. This is similar to the natural interactions performed when taking a card from a stack of blank cards and placing it where it can easily be edited. Once the new card is created, its information can be revised via the direct edit feature (Figure 4). MasePlanner also uses a similar approach for creating iteration and backlog regions.

\subsection{Iteration Planning}

Organizing story cards in MasePlanner is similar to organizing story cards in a natural tabletop setting. Story cards, iterations, and backlogs are all represented by colored artifacts that are easily moved with minimal use of the mouse. This is similar to grabbing a pile of story cards, in a physical environment, and moving them across the table.

\footnotetext{
2 AgilePlanner's handwriting recognition approach will be integrated with MasePlanner this summer.
}

Moving a story card from one iteration/backlog to another involves simply dragging it from one region to the other. This is again similar in effort to picking up a card from a given location on the table and placing it at another. Supporting this method of interaction allows for team members to interact more naturally with the story cards.

MasePlanner uses the location of story cards to assign a priority and allows for iteration estimate and actual effort tracking. Team members can prioritize cards by dragging higher priority cards to the top of the iteration or backlog regions. Priorities are also indicated on the cards by a numeric value (Figure 4). Time estimates and actual effort for all iterations are calculated based on the estimates and actual effort for its containing story cards and are summarized at the top of the iteration region (Figure 4). This provides the accounting features that exist in many non-card based planning tools.

Updating of planning data in MasePlanner is performed immediately following a change to a field or location. This update sends new data to the server, which in turn sends the updates to other clients subscribing to the same project. This method of updating allows for multiple clients to receive changes as close to real time as possible.

\section{Future Directions}

MasePlanner remains under development and as such has not been evaluated. In this section, we present our next steps.

\subsection{Improving the Tool}

The previous section highlighted the current abilities supported by MasePlanner. However, in its current state, it does not support any type of mouse awareness from other clients. Extending MasePlanner to support telepointers [12] will allow for connected clients to gesture via their mouse in the workspace. Supporting gestures in a distributed planning environment would allow for further enhancements to communication and allow for an environment that further simulates collocated collaboration.

To support natural story card creation, entering information directly on the story card is necessary. To augment this input method we are working on supporting pen-based inputs [6]. The combination of both of these approaches will allow collaborators to interact in a way that is convenient and easy.

Tabletop computing is quickly catching the attention of collaborative teams as it allows for increased face-to-face collaboration. Extending 
MasePlanner, by incorporating ideas from AgilePlanner, to support tabletop environments, would allow for an enhanced planning experience. Considerations like orientation [13] and simultaneous user interaction [14] will be necessary.

Combining all of the above mentioned features will create a distributed planning environment that closely simulates natural planning environments.

\subsection{Evaluating the Approach}

In evaluating the effectiveness of MasePlanner, it will be necessary to conduct observational studies comparing the interactions of the participants using MasePlanner in relation to traditional card-based planning.

An observational study is being planned to investigate student teams as they complete a multiiteration project. The study will be broken down into three phases, all of which will have some team members not physically present at the meeting. First teams will conduct a planning meeting using paper based planning methods. Second teams will use an existing planning tool, such as MASE and the third and final phase will use MasePlanner.

Participants will be asked to fill out questionnaires regarding their experiences following each planning meeting. This feedback will be used to determine if the methods supported communication and collaboration amongst all participants and to provide insight into ways of improving MasePlanner.

\section{Conclusions}

This paper presents a card-based planning tool to allow for distributed agile planning by supporting natural interactions with planning artifacts.

Existing planning tools allow for some interaction between distributed team members in addition to the ability to monitor estimate calculations, and the prioritization of stories. They are limited in their ability to support natural interactions for distributed teams. MasePlanner is an attempt to merge the benefits of existing distributed planning tools with the benefits of collocated card-based planning.

This work is still in progress and has not been evaluated. We hope that through formal evaluation we will be able to determine the usefulness of MasePlanner and to highlight potential usability concerns. We are hopeful that these results will spawn further examination and research into the realm of distributed card-based project planning.

\section{References}

[1] K. Beck, Extreme Programming Explained: Embrace Change, Addison Wesley, Boston, 2000

[2] F. Maurer, "Supporting Distributed Extreme Programming" Proceedings of XP/Agile Universe 2002, Illinois, USA, 2002, pp. 13-22

[3] VersionOne LLC. (2006). Agile Project Management Tool. [Online] Viewed 2006, March, 10. Available: www.versionone.net/products.asp

[4] Rally Software Development Corp (2006) Software Development Life Cycle Management for Agile Development Teams. [Online]. Viewed 2006, March, 10. Available: www.rallydev.com/products.jsp

[5] XPlanner. (2006). XPlanner Overview. [Online] Viewed 2006, March, 17. Available: http://xplanner.org

[6] L. Liu, H. Erdgmous, F. Maurer, "An environment for collaborative Iteration Planning", In proceedings of Agile 2005, Colorado, 2005,

[7] Danube Technologies Inc. (2006). ScrumWorks - Product Overview. [Online]. Viewed 2006, March, 10. Available: http://danube.com/scrumworks

[8] S. Pinna, S. Mauri, P. Lorrai, M. Marchesi, N. Serra, "XPSwiki: An Agile Tool Supporting the Planning game", Proceedings of XP 2003, Italy, 2003, pp. 104-113

[9] L. Liu "An Environment for Collaborative Agile Planning", M.S. Thesis, University of Calgary, Calgary, AB, Canada, 2006

[10] Eclipse Foundation. (2006). Eclipse. [Online]. Viewed 2006, March, 6. Available: www.eclipse.org

[11] Eclipse Foundation. (2006). Graphical Editing Framework. [Online]. Viewed 2006, March, 8. Available: www.eclipse.org/gef/

[15] S. Greenberg, C. Gutwin, M.Roseman, "Semantic Telepointers for Groupware", Proceedings of Australian Conference on Computer-Human Interaction, New Zealand, 1996, pp. 54-61

[13] R. Kruger, S.Carpendale, S.D. Scott, S. Greenberg, "Roles of orientation in tabletop collaboration: comprehension, coordination and communication", Journal of Computer Supported Collaborative Work, Netherlands, 2004, pp.501-537

[14] S.D. Scott, K.D. Grant, R.L. Mandryk, "System Guidelines for Co-located, Collaborative Work on a Tabletop Display", Proceedings of European Conference Computer Supported Cooperative Work, Finland, 2003, pp. 159-178 